\title{
Processing and Querying Description Logic Ontologies Using Cartographic Approach
}

\author{
Krzysztof Goczyła, Teresa Grabowska, Wojciech Waloszek, Michał Zawadzki \\ Gdańsk University of Technology, \\ ul. Narutowicza 11/12, 80-952 Gdańsk, Poland \\ \{kris, tegra, wowal, michawa\}@eti.pg.gda.pl
}

\begin{abstract}
Description Logic (DL) is a formalism for knowledge representation that recently has gained widespread recognition among knowledge engineers. After a brief introduction to $D L$, the paper presents a $D L$ reasoner developed at Gdansk University of Technology in the course of a EU $6^{\text {th }} \mathrm{FP}$ project PIPS. The reasoner is based on an original idea called Knowledge Cartography. The paper presents basics of Knowledge Cartography, its potentials and limitations, and compares the solution with other known DL reasoners. Also, some results of preliminary exploitation of the reasoner are discussed.
\end{abstract}

1. Introduction. Description Logic (in short: DL) is a formalism for knowledge representation that is based on the first-order logic [1]. DL has recently gained widespread popularity among knowledge engineers, mainly due to the fact that OWL-DL [2], the language for Semantic Web ontologies [3, 4] promoted by W3C, is based on a Description Logic dialect. During the past several years a number of DL reasoners have been developed, the most prominent being Racer and FaCT. Each of these reasoners has its peculiarities and limitations (e.g. $\mathrm{FaCT}$ is not able to process ontologies with individuals; while Racer turns out not to be able to process complex ontologies). In the paper we present another DL reasoner developed in the course of a EU $6^{\text {th }}$ FP project PIPS (Personalized Information Platform for Life and Health Services, [5]). One of major components of the PIPS system is a Knowledge Management Subsystem (KMS) [6] that is aimed at performing advanced inference services from OWL-DL ontologies for other layers of PIPS.

The approach we applied in our reasoner is quite different from the approaches in other DL reasoners. In this approach (primarily proposed by W. Waloszek, [7]), dubbed as Knowledge Cartography, we treat a universe as a map of overlapping concepts. Each concepts consists of a number of atomic regions in the map. Each region is assigned a unique bit position in a string of bits called a signature of a concept. In that way, we reduce inference problems to appropriate Boolean operations on signatures.

The rest of the paper is organized as follows: In Section 2 we give a brief introduction to DL and related inference problems. In Section 3 we introduce ideas of Knowledge Cartography. In Section 4 we discuss some implementation details of the approach in KASEA, a knowledge inference engine for a Knowledge Management Subsystem in PIPS. In Section 5 we conclude the paper with further work.

2. Ontologies and Description Logics. This section is devoted for those of readers who are not familiar with ontologies and Description Logic itself. We give a short introduction "by example"; for a comprehensive study an interested reader is referred to [1].

2.1. Ontologies. Informally, an ontology is a description of terms that are important for a given domain of interest. An ontology can be viewed as an encyclopedia or a dictionary where we can find knowledge on some subject. Being a bit more formal, an ontology is an explicit (i.e. expressed in a formal language) specification of conceptualization. The "conceptualization" is meant as a model of some part of world, expressed in terms of concepts, their properties and relations between the concepts. In this definition, the term "concept" appears that is of crucial importance for understanding ontologies. We will specify it more precisely in the following. 
The first ontology-based method of knowledge representation in computer science were frames proposed by Minsky [8]. According to Minsky, any entity in the world is a named frame, each containing slots (properties) and facets (constraints). Slots and facets are also frames. Most important frames are classes (called also concepts). Classes can have instances (that are frames too) that represent individuals or other classes. In Fig. 1 there are two frames: a class frame named DOG and an instance frame named MAX. Note that SLOT1 of MAX has a standard name INSTANCE-OF with a predefined meaning.

\begin{tabular}{|l|l|}
\hline NAME & DOG \\
\hline SLOT1 & SKIN: HAIR \\
SLOT2 & BORN: VIVIPAROUS \\
SLOT3 & GENDER: MALE OR FEMALE \\
SLOT4 & VOICE: BARKING \\
SLOT5 & LEGS: CARDINALITY: 4 \\
\hline
\end{tabular}

\begin{tabular}{|l|l|}
\hline NAME & MAX \\
\hline SLOT1 & INSTANCE-OF: DOG \\
SLOT2 & SKIN: HAIR \\
SLOT3 & BORN: VIVIPAROUS \\
SLOT4 & GENDER: MALE \\
SLOT5 & VOICE: BARKING \\
SLOT6 & LEGS: CARDINALITY: 4 \\
\hline
\end{tabular}

Fig.1 A class frame and an instance frame.

Frames are a very general mechanism. For example, an instance of a class may be also a class, which leads to a notion of metaclasses. Frames have no single precise formalism, which makes difficult to construct reasoners. However, some notions from frames theory appear in modern object-oriented software and knowledge engineering, also in Description Logic.

Description Logic (DL) is a formalism (or rather - a family of formalisms) based on First Order Logic (FOL). DL has both precise formalism and easy to understand set-theory interpretation. For these reasons it has recently become a commonly accepted basis for knowledge representation and management systems. Actually, the OWL-DL language, promoted by W3C within the Semantic Web initiative [3] is based on a DL dialect.

The approach to representing knowledge about world as a DL ontology is based on the following three natural and simple assumptions:
1. There is a universe (domain of interest) that is to be specified as an ontology.

2. The universe consists of individuals that are instances of concepts.

3. Concepts are related to each other by binary relations called roles.

According to the above, a DL ontology consists of two parts: a terminological part (TBox) and an assertional part (ABox). TBox contains concepts, roles and axioms that define constraints on concepts and roles. ABox contains instances of concepts (in the form of unary assertions) and instances of role (in the form of binary assertions). An ontology does not have to have both parts. Very often, ontologies contain only a TBox, although one can also imagine an ontology with ABox only, with concepts and roles given implicitly.

DL ontologies are data for DL knowledge bases (KB). For ontologies to be processed and reasoned over, a KB must be equipped with a reasoner, or inference engine. There also must exist a language to express the ontology. The required components of a DL knowledge base are depicted in Fig. 2.

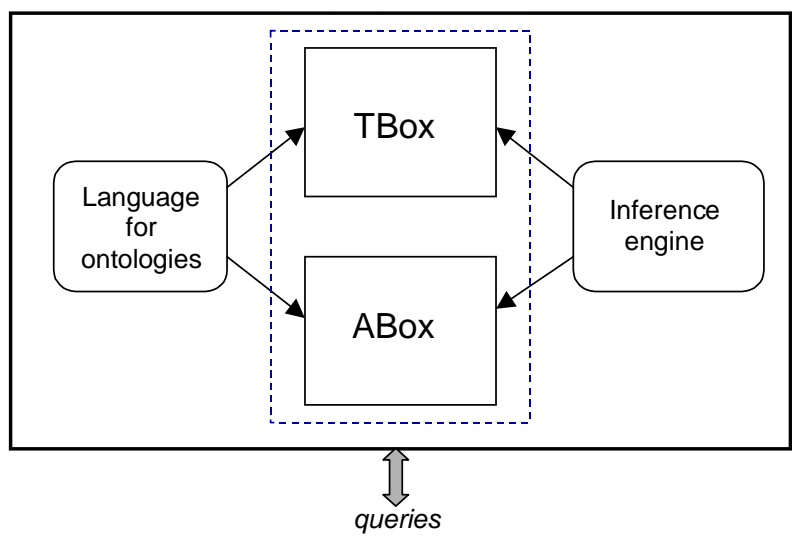

Fig. 2. A DL knowledge base.

Any language from a rich family of DL languages has basic components. They are:

- atomic concepts, including the universal concept $\mathrm{T}$ (Top) that represents the universe, and the empty concept $\perp$ (Bottom) that is a concept that cannot have any instances;

- atomic roles;

- constructors that are used to create complex concepts and roles.

In this paper we assume that ontologies are specified in $\mathcal{A L C}$ - a DL dialect that is simple 
enough for an introduction of DL, yet powerful enough to define non-trivial ontologies. Table 1 presents constructors of the language (note that in $\mathcal{A L C}$ there are no constructors for roles).

\begin{tabular}{|l|l|}
\hline$\neg C$ & Negation of a concept \\
\hline$C \sqcap D$ & Intersection of two concepts \\
\hline$C \sqcup D$ & Union of two concepts \\
\hline$\exists R . C$ & $\begin{array}{l}\text { Existential quantification: a set of } \\
\text { individuals that are at least once related by } \\
\text { role } R \text { to an individual from concept } C\end{array}$ \\
\hline$\forall R . C$ & $\begin{array}{l}\text { General quantification: a set of individuals } \\
\text { for which all (possibly zero) relations by } \\
\text { role } R \text { are with individuals from concept } C\end{array}$ \\
\hline
\end{tabular}

Table 1. Constructors of $\mathcal{A L C}$.

2.2. Inference problems. In the following, an exemplary ontology of family relations will be used (see Table 2). The ontology contains concepts Person, Man, Woman, Parent, Father and Mother, and role hasChild. TBox of the ontology contains also axioms expressed with two operators: concept equivalence $(\equiv)$ and concept inclusion ( $\sqsubseteq$ ), with obvious meaning.

\begin{tabular}{l|l}
\hline TBox & Man $\sqsubseteq$ Person \\
Woman $\sqsubseteq$ Person \\
Woman $\sqcap$ Man $\equiv \perp$ \\
Parent $\equiv$ Person $\sqcap \exists$ hasChild.Person \\
Father $\equiv$ Man $\sqcap$ Parent \\
Mother $\equiv$ Woman $\sqcap$ Parent \\
\hline ABox & Woman (Ann) \\
& Woman (Jane) \\
& Man (Charles) \\
hasChild (Ann, Jane) \\
hasChild (Ann, Charles) \\
Woman (Ann)
\end{tabular}

Table 2. An exemplary ontology.

The axioms of the ontology state that a Man and a Woman are Persons, and no one can be simultaneously a Man and a Woman, a Parent is a Person that has at least one human child, a Father (or a Mother) is a Parent that is a Man (or a Woman). ABox contains some known facts about Ann, Jane and Charles who are members of the family (e.g. Jane and Charles are children of Ann). We will use the above ontology to explain basic DL inference problems.

Subsumption: Concept $C$ subsumes concept $D$ (denotation: $D \sqsubseteq C$ ) if the set of instances of $D$ is always a subset of the set of instances of $C$. In our example, some subsumptions are given explicitly as axioms. Other ones can be inferred, e.g. Father $\sqsubseteq$ Person and Mother $\sqsubseteq$ Person.

Satisfiability: Concept $C$ is satisfiable if it can have instances. In other words, concept $C$ is unsatisfiable if $C \equiv \perp$. In our example, all the concepts are satisfiable. If we, however, add to TBox a new concept Fosterer and an axiom Fosterer $\equiv$ Mother $\sqcap$ Father, then Fosterer is unsatisfiable.

Disjointness: Concepts $C$ and $D$ are disjoint if sets of their instances are always disjoint $(C \sqcap D$ $\equiv \perp)$. In our example, pairs of concepts: (Woman, Man) and (Mother, Father) are disjoint.

Equivalence: Concepts $C$ and $D$ are equivalent $(C \equiv D)$ if sets of their instances are always equal.

The above inference problems all refer to TBox. It is easy to show that they are not independent of each other. For instance, checking if two concepts are disjoint is equivalent to checking satisfiability of their intersection. Indeed, all the above problems can be reduced to subsumption [1].

Existence of ABox leads to other basic inference problems that refer to the whole ontology. Below we use a self-explanatory functional notation of DIG interface [9].

Instance retrieval: Retrieve from a knowledge base all individuals that are instances of a given concept. (denotation: instances $(C)$ ). For example, a response to instances (Mother) is a singleton set $\{$ Ann $\}$.

Instance check: Check if a given individual is an instance of a given concept (denotation: instance $(x, C)$ ). For example, the response to instance (Ann, Mother) is true.

Consistency check: A knowledge base $K$ is consistent (denotation: consistent $(K)$ ) if all the concepts from TBox are satisfiable and ABox does not contain any false individual (an 
individual is said to be false if it is not an instance of any concept other than $\perp$ ). Our exemplary knowledge base is consistent. But let us assume that we add to ABox two new assertions: Woman(Mary), Man(Mary). After that the knowledge base has lost its consistency because Mary must be an instance of the empty concept $\perp$, which is absurd.

Now we extend our exemplary ontology by a role hasSon (with its natural meaning). It is clear that having a son implies having a child, so we can formulate the following axiom:

$$
\text { hasSon } \sqsubseteq \text { hasChild }
$$

But how to express that a son (i.e. the right-hand side filler of hasSon) is a Man? In other words, how to define the range of role hasSon? We can do it in the next axiom:

$$
\exists \text { hasSon. } \neg \text { Man } \equiv \perp
$$

which can be interpreted as follows: if any individual has a son who is not a Man, then this individual is false (i.e. it does not exist). Similarly, we can state that only Parents may have sons (i.e. define the domain of a role):

$$
\exists \text { hasSon. } T \sqsubseteq \text { Parent }
$$

which can be read as a statement that if any individual has any son, then this individual is a Parent.

Having defined the domain and the range of hasSon, we add to ABox a new fact:

$$
\text { hasSon (Charles, John) }
$$

Now let us issue against our knowledge base two queries: types(John) and types(Charles). (The types $(x)$ query asks a KB for all concepts from TBox that $x$ is an instance of.) Because Charles is not a false individual, John must be a Man (a member of the range of hasSon), and also a Person (any Man is a Person). Hence, the response to types(John) is \{Man, Person\}. The response to types(Charles) is \{Parent, Man, Person $\}$, which is derived from the axiom defining the domain of hasSon and from explicitly given contents of ABox and TBox.

Traditional approaches to solving the inference problems exploit different kinds of structural analysis of terminology (structural subsumption) or so-called tableau algorithms [1]. In general, inference problem are of exponential time/space complexity, so it is of crucial importance for real-life applications to optimize algorithms used so that to obtain acceptable performance of an inference engine. These issues will be discussed in Section 4 .

2.3. The Open World Assumption. Now we go back to our original exemplary ontology from Table 2. Consider a query instances( $\neg$ Mother). It might seem that the response to this query is a two-element set \{Jane, Charles\}. However, the proper (in the sense of DL reasoning) response is only $\{$ Charles $\}$. It is due to the fact that DL knowledge bases conform to the Open World Assumption (OWA). According to OWA, the knowledge that a knowledge base has is incomplete: there may be other true assertions about the world that the $\mathrm{KB}$ is not aware of because they have not been stored in ABox yet. So, it is still possible that Jane is a Mother (nothing in the KB contradicts this). On the other hand, the KB "knows" for sure that Charles is not a Mother, because he is a Man (which is disjoint with a Woman and, consequently, with a Mother).

But what should our $\mathrm{KB}$ respond to the following query: instance(Jane, Mother)? The proper answer is don't know: the $\mathrm{KB}$ has no indication that Jane is a Mother and no indication that Jane is not a Mother. This leads to three-valued logic in KBs: true, false and don't know. (Note, however, that most known DL inference engines would respond false to both queries: instance(Jane, Mother) and instance(Jane, $\neg$ Mother), leaving appropriate interpretation of these to the user.)

OWA fundamentally distinguishes knowledge bases from databases. Databases conform to the Closed World Assumption (CWA): any query issued against a database concern only those data that are explicitly stored in the database (and any SQL executor works in this way). Strictly conforming to OWA in knowledge bases may sometimes lead to confusion among "typical" users who tend to treat a knowledge base as a bit "wiser" database, just equipped with capabilities that enable to infer new facts from the stored ones. Consider, for example, a query about the number of Ann's children. If we stored the assertions from Table 2 in a database and issued a corresponding SQL query, the answer would be 2 . But what if the same query is issued against our exemplary KB? The answer could be "2 or more" or similar. Indeed, there may be other assertions of form 
hasChild(Ann, $y$ ) that are true but not yet stored in the KB.

This and similar responses may sound strange for a user whose intention is to learn the number of known Ann's children or-going back to the above example with mothers and non-mothers - to find out which persons are not known to be mothers. In the first case a user would expect the answer "2", and in the second case the answer \{Jane, Charles\}. For these reasons, some ways of "closing" the KB world are introduced. One of them is the epistemic operator K. Query that contains this operator relates to facts that are known to a knowledge base in its current state. For example, consider the query $\neg$ Kinstances(Mother) which asks for all individuals that are not known to be a Mother. The response would be \{Jane, Charles\}, as expected, because the $\mathrm{KB}$ does not know if Jane has any children.

The $\mathbf{K}$ operator is one of modal operators that are used to express different conditions (modalities) and constraints concerning meaning of information stored in a KB. A thorough overview of other modal operators is in [1].

2.4. Applications. DL ontologies, usually specified in OWL-DL, are presently vigorously developed within the Semantic Web Activity framework. They are used as a means of interchange of information, or knowledge, among web-enabled information systems, to attain maximal interoperability between them. One of such systems is PIPS (Personalized Information Platform for Life and Health Services [5]), currently under development within a $6^{\text {th }}$ EU Framework Programme, Priority "e-health" (Contract No 507019). One of its elements is a knowledge base that processes OWL-DL ontologies concerning areas of medicine, nutrition and healthy life-style. The PIPS KB is managed by KASEA, a system for processing and reasoning over DL ontologies, based on a novel idea of Knowledge Cartography. The rest of the paper is a presentation of Knowledge Cartography.

3. Knowledge Cartography. The idea of Knowledge Cartography is based on the following assumptions:

1. TBox component of the knowledge base is modified so rarely that actually it may be considered constant.
2. A knowledge base is queried much more often than it is updated (by update we mean here addition of new assertions into ABox). Therefore, performance of retrieval is crucial, while performance of updates is not.

3. A knowledge base should be able to store and efficiently process numerous individuals.

The Knowledge Cartography and algorithms behind it aim at storing in a knowledge base as many conclusions (inferred facts) about concepts and individuals as possible. Any such conclusion can be quickly retrieved in the process of query answering and remains always valid due to the fact that the terminology is not changed. By appropriate organization of a knowledge base identical conclusions can be applied to many individuals, which facilitates efficient information retrieval and reduces the size of a database that is used to store the knowledge base.

3.1. The general idea. Knowledge Cartography takes its name after a notion of "a map of concepts". A map of concepts is basically a (graphical and symbolic) description of relationships that hold between concepts in a terminology, seen as sets of individuals. The map is created during the knowledge base creation. The map of concepts can be graphically represented as a Venn diagram (see Fig. 3). Each atomic area of the map (i.e. an area that does not contain any other area; called henceforth a region) represents a single valid intersection of concepts. By valid we mean an intersection that is satisfiable with respect to a given terminology. Unsatisfiable regions (not allowed by terminological axioms) are excluded from the map (as in Fig. 3b, where two additional axioms excluded four regions from the map). The algorithm for processing ontologies, called Cartographer, calculates a number of valid regions $n$ and assigns each region a subsequent integer from the range $[1, n]$ (shown in Fig. 3 as numbers within regions). Because any area in the map consists of some number of regions, any area can be represented by a string of binary digits (bits) of length $n$ with " 1 "s at positions corresponding to contained regions and " 0 "s elsewhere.

According to this rule, any concept in a terminology is assigned a signature - a string of bits representing the area covered by the concept in the map. It is important that in this way we 
a)

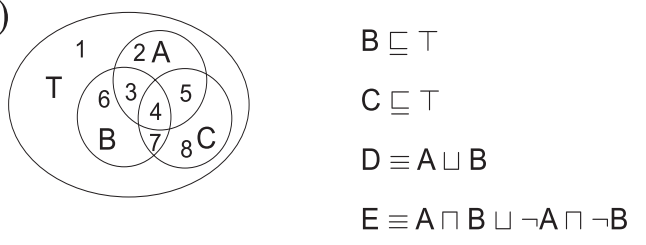

Signatures:

$\begin{array}{ll} & 12345678 \\ \text { A } & 01111000 \\ \text { B } & 00110110 \\ \text { C } & 00011011 \\ \text { D } & 01111110 \\ \text { E } & 10110001\end{array}$ b)

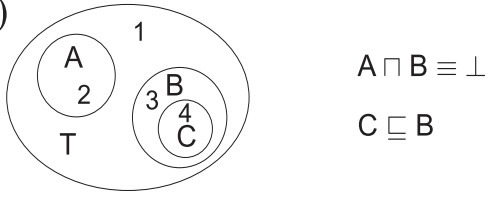

Signatures:

A 0100

B 0011

C 0001

D 0111

E 1000

Fig. 3. An example of a map of concepts (a) with two terminological axioms added (b).

can represent any combination of complement, union and intersection of any concepts by simply performing Boolean negation, disjunction and conjunction.

Formally speaking, we define a function $s$ from concepts in TBox to elements of a Boolean algebra $\mathbf{B}^{n}=\{0,1\}^{n}$ (the set of bit strings of length $n$ ). It can be shown the only requirement that should be met by function $s$ so is:

$$
s(C) \leq s(D) \Leftrightarrow C \sqsubseteq D
$$

The $\leq$ operator is understood in terms of a Boolean algebra (i.e. a bit string that includes all " 1 "s of another string at the same positions is greater than or equal to the other string). In any such a function regions are mapped to atoms of $\mathbf{B}^{n}$, i.e. strings of " 0 "s with a single " 1 ". Moreover, for any concepts $C$ and $D$ the following equalities hold:

$$
\begin{aligned}
& s(\neg C)=\neg s(C) \\
& s(C \sqcap \mathrm{D})=s(C) \wedge s(D) \\
& s(C \sqcup \mathrm{D})=s(C) \vee s(D)
\end{aligned}
$$

Having determined $s$, any basic TBox reasoning problem can be solved by appropriate operations on signatures. Indeed:

- A query for equivalence of concepts $C$ and $D$ can be performed by checking whether $s(C)=$ $s(D)$.

- A query for subsumption of concepts $C$ and $D$ can be performed by checking whether $s(C) \leq$ $s(D)$.

- A query for disjointness of concepts $C$ and $D$ can be performed by checking whether $s(C) \wedge$ $s(D)=\{0\}^{n}$.
Also advanced reasoning problems, like least common subsumer problem or most specific concept problem, can be solved by simple operations on signatures.

From (1) we deduce that the order of the range of function $s$ should be equal to the number of terminologically unequivalent concepts that can be expressed in the terminology. With introduction of $\exists R . C$ and $\forall R$.C constructs this number reaches infinity (namely $\aleph_{0}$ ), because existential and general quantifiers can be nested to theoretically unlimited level (for instance, a concept in TBox can be defined as $\exists R . \exists R . \exists R . \ldots \exists R . C$ ).

Due to this fact, we have made an important decision restricting the use of $\exists R$.C and $\forall R$.C constructs in queries. The only concepts of the form $\exists R$.C and $\forall R$.C that can be used in queries are those explicitly defined in the terminology. In this way we limit the number of unequivalent concepts, making possible to fix the length of signatures. This restriction limits expressiveness of ad hoc queries accepted by the system, but our experiences gained from using KASEA in real-life (although still experimental) environment show that this limitation is not severe for knowledge base users.

Analogical techniques as for TBox can be applied to reasoning over ABox. We assign each individual $a$ in ABox a signature of the most specific concept the individual is an instance of (we denote this concept as $C_{a}$; this concept need not be defined explicitly in TBox). After determination of signatures for all individuals in a $\mathrm{KB}$ we can reduce all ABox reasoning problems to TBox reasoning problems, which in 
turn can be solved by operations on signatures. For example, checking if individual $a$ is an instance of concept $C$ is reduced to checking whether concept $C_{a}$ is subsumed by concept $C$. And checking consistency of a knowledge base consists in simply checking if there exist in the $\mathrm{KB}$ any concept or any individual whose signature is equal to $\{0\}^{n}$.

3.2. The map creation algorithm. The main algorithmic problem in Knowledge Cartography is determination of function $s$, i.e. creation of a map of concepts. We can formulate this problem as follows: Given an $\mathcal{A L C}$ terminology $\mathcal{T}$, for each atomic concept and each concept of the form $\exists R$.C (called jointly mapped concepts) generate its signature. (Note that concepts of the form $\forall R$.C do not have to be considered because they can be converted to the equivalent form of $\neg \exists R$. $\neg C$.) The problem is not polynomial (unless $\mathrm{P}=\mathrm{NP}$ ) because it can be reduced to the problem of CNF-satisfiability. However, some optimization techniques can make the process of map (and signatures) creation efficient for reallife ontologies.

The process of creation of signatures is based on the fact that regions of the map are mapped to the atoms of $\mathbf{B}^{n}$ that constitute the range of function s. Regions can be viewed simply as valid intersections of all mapped concepts in $\mathcal{T}$ or their complements, i.e. all possible complex concepts of the following form:

$$
L_{1} \sqcap L_{2} \sqcap \ldots \sqcap L_{k}
$$

where $k$ is the number of mapped concepts and $L_{i}$ is a placeholder for $i$-th mapped concept or its complement. Using this approach we may see a terminology as a set of first-order logic formulae, mapped concepts being variables, and reduce the problem of a map creation to finding a truth table for the terminology. Each satisfiable combination of variable values may be treated as a region.

From among many techniques available we applied Ordered Binary Decision Diagrams (OBDD) originally proposed in [10]. Cartographer systematically builds an OBDD tree for the whole terminology by combining (using the logical AND operation) a current tree with trees generated for formulae corresponding to consecutive axioms. The initial tree is a tree with a single node (OBDD for a tautology).
Axioms are converted into first-order logic formulae as proposed in [10], but the method is somehow simplified because concepts of the form $\exists R$.C (or $\forall R$.C) are represented as one variable. Each new mapped concept is assigned a new variable name. For example, the axiom:

$$
\begin{aligned}
\text { Momo } \equiv & \text { Person } \sqcap \forall \text { hasChild.Man } \sqcap \\
& \exists \text { hasChild.Man }
\end{aligned}
$$

is converted to the equivalent form:

$$
\begin{aligned}
\text { Momo } \equiv & \text { Person } \sqcap \neg \exists \text { hasChild. } \neg \text { Man } \sqcap \\
& \exists \text { hasChild.Man }
\end{aligned}
$$

and subsequently to the formula:

$$
c_{1} \leftrightarrow c_{2} \wedge \neg e_{1} \wedge e_{2}
$$

An outline of the algorithm is presented below:

Input: A terminology $\mathcal{T}$ expressed in $\mathcal{A} \mathcal{L C}$.

Output: An OBDD tree $T$ for terminology $\mathcal{T}$.

Initialize $T$ to OBDD of any tautology.

For each axiom $A$ from $\mathcal{T}$ :

Convert $A$ to the formula $F$ in the way described above.

Generate the OBDD $U$ for the formula $F$. $T:=T \wedge U$ (where $\wedge$ denotes conjunction of two OBDD trees in the sense of [10])

Next

Direct application of the above algorithm to some terminologies may lead to generation of spurious (unsatisfiable) regions, as shown in Fig. 4a. According to the axioms added to the terminology, an individual cannot belong to $\exists R . B$ and not belong to $\exists R . A$ (because, according to the inclusion axiom, each member of $B$ is a member of $A$ ). In order to exclude the spurious regions we perform some postprocessing that produces a tree $T^{\prime}$ on the basis of $T$. The post-processing consists in checking whether for each combination of values of variables $e_{i}$ that concern the role $R$ and are satisfiable with respect to $T$ it is possible to create a set of individuals that would satisfy the combination. For instance, in Fig. 4a the following atomic intersection is satisfiable with respect to $T$ :

$$
\neg A \sqcap \neg B \sqcap C \sqcap \neg \exists R . A \sqcap \exists R . B
$$


a)

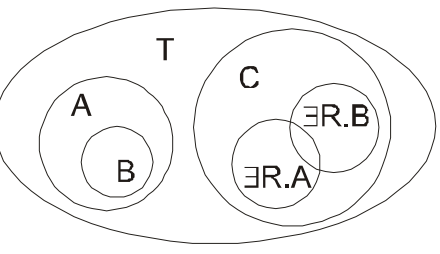

b)

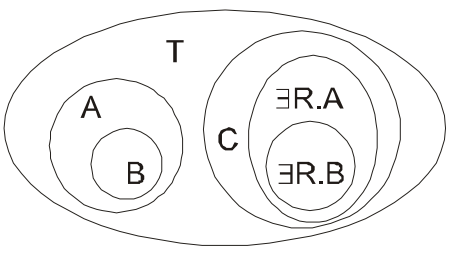

$\mathrm{A} \sqcap \mathrm{C} \equiv \perp$

$B \sqsubseteq A$

$\exists$ R.A $\sqsubseteq C$

$\exists \mathrm{R} . \mathrm{B} \sqsubseteq \mathrm{C}$

Fig. 4. A map of concepts before post-processing (a) and after post-processing (b).

This region (represented in Fig. 4a as the part of $\exists R . B$ that is not contained in $\exists R . A$ ) should be, however, excluded from $T^{\prime}$ during postprocessing because the following family of concepts:

$$
\neg A \sqcap B \sqcap \ldots
$$

is not satisfiable with respect to $T$ (because there cannot exist any individual that belongs to $B$ and does not belong to $A$ ).

Details of post-processing are outside the scope of this paper. We have actually shown that the post-processing phase is sound and complete, i.e. all intersections it excludes are invalid and there is no invalid intersection it does not exclude.

4. The KASEA system. Cartographic Approach has been successfully applied in the prototype of the PIPS system. The Knowledge Inference Engine (KIE), a vital component of KASEA, uses Cartographer to load PIPS ontologies and to infer and store inferred facts. KIE allows for processing a terminology (TBox) and assertions (ABox). Data are stored in a relational database (PostgreSQL 8.0), which allows for using builtin optimization techniques like indexing or query optimization. In the following subsections we present some query processing scenarios and performance issues.

4.1. Query-processing. Knowledge base updates (Tells) and queries (Asks) are formulated in the DIGUT language [11], an interface based on DIG/1.1 especially modified for querying KASEA. Tells that can be handled by KASEA are concept assertions of the form $C(a)$ and role assertions of the form $R(a, b)$. Due to assumptions stated at the beginning of Sec. 3, KASEA does not handle Tells that concern terminological axioms.

For processing a concept assertion $C(a), C$ has to be an expression built of concepts used in the terminology. Constructs of the form $\exists R . A$ are allowed only if a signature for this construct has been determined during loading the terminology into the knowledge base. The course of actions consists of the following steps:

1. Calculate a signature $s(C)$.

2. If $a$ is not in the database, add it to the database and assign it a signature $s(C)$. STOP.

3. Otherwise combine the signature of $C_{a}$ with $s(C)$ using logical AND operation.

4. If a signature of $C_{a}$ has been changed, update the neighborhood of $a$ (see below). STOP

In processing of a role assertion of the form $R(a, b)$ only neighborhood update is performed.

Necessity of updating neighborhood is a consequence of the fact that changing our knowledge about membership of an individual $a$ may change our knowledge about individuals related to $a$. In the current version of KASEA simple mechanisms of positive role checking and negative role checking has been applied. In the positive role checking every pair $(a, b)$ related with role $R$ is checked against all mapped concepts of the form $\exists$ R.C. If $b$ is a member of the concept $C$, the signature of $C_{a}$ is combined with $s(\exists R . C)$ using logical AND operation (because $a$ has to be a member of the concept $\exists$ R.C). In the negative role checking every pair $(a, b)$ related with $R$ is also checked against all mapped concepts of the form $\exists R$.C. If $a$ is a member of the concept $\neg \exists R$.C, the signature of $C_{b}$ is combined with $s(\neg C)$ using (because $b$ may not be a member of $C$ ).

This process is recursively repeated if the signature of any individual has been changed. The process eventually stops after a finite number of steps because the number of individuals in $\mathrm{ABox}$ is finite and in each update the number of " 1 "s in signatures of individuals being processed may only decrease. Potentially, 
a)

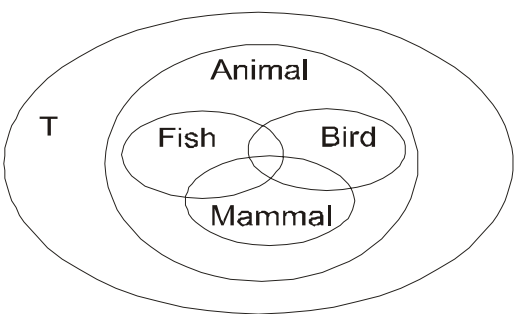

b)

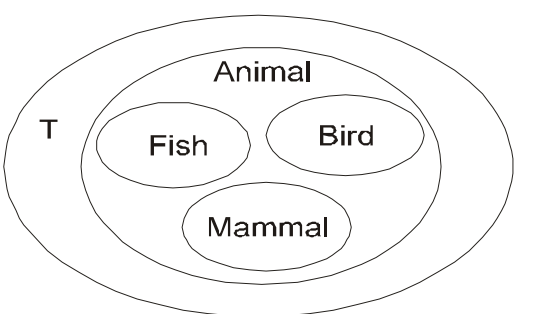

Fig. 5. Division of a domain space for a terminology: a) without disjoints, and b) with disjoints.

the process of neighborhood update is prone to combinatorial explosion, but in real-life ontologies there is little chance for that. We are working on another way of updating ABox.

Most Asks can be brought down to subsumption checking and, consequently, to simple operations on signatures. For example, the instance retrieval problem for a concept $C$ can be performed as finding all individuals $a$ such that $C_{a}$ is subsumed by $C$. During subsumption checking some preliminary checks are used to quickly exclude existence of the subsumption relation (e.g. counting "1"s in segments of signatures). Then bitwise Boolean operations are performed in order to check whether two signatures are in the $\leq$ relation.

4.2. Performance results. As for other inference algorithms, the space and time complexity of processing a terminology by Cartographer is in the worst case exponential. Indeed, the maximum number of regions that the space can be divided into by $k$ concepts is $2^{k}$, which results in signatures of exponential length. The corresponding terminology would consist of $k$ concepts not related to each other at all. Note that such a case is very rare in practical terminologies (if found at all). Specifically, this would mean that any subset of concepts may have instances, because no pair of concepts is declared disjoint.

As an example, consider a terminology containing one root concept Animal and three direct subconcepts: Bird, Fish, and Mammal (axioms 1, 2, and 3 below):
1. Bird $\sqsubseteq$ Animal
4. Bird $\sqcap$ Fish $\equiv \perp$
2. Fish $\sqsubseteq$ Animal
5. Bird $\sqcap$ Mammal $\equiv \perp$
3. Mammal $\sqsubseteq$ Animal
6. Fish $\sqcap$ Mammal $\equiv \perp$

In such a terminology, Bird, Fish and Mammal are unrelated, which means that we could declare one individual to be simultaneously a Bird, and a Fish, and a Mammal. Such a terminology would create a domain space with 9 regions (Fig. 5a). Let us add three disjoints (axioms 4, 5, and 6 above). The number of regions in the domain space drops to 5 (Fig. 5b). Actually, we have now a pure tree taxonomy, which reflects reality among species of animals.

It is easy to show that for pure tree taxonomies the signature size grows linearly with $k$. Our experiments show that the observed time complexity of map generation algorithm is proportional to $k \log k$ for such taxonomies except for inherently quadratic $\left(\mathrm{O}\left(k^{2}\right)\right)$ signature generation phase (the algorithm generates $k$ signatures of length proportional to $k$ ). The results of terminology processing time are presented in Table 3 (all tests were performed on a PC with Celeron $2.4 \mathrm{GHz}$ and $512 \mathrm{MB}$ RAM). It is worth to notice that the time is independent on the order of axioms in terminologies as special heuristics have been used to estimate the best possible ordering.

The ABox queries processing performance of KASEA has been tested and compared with several freely available tools: Jena $2[12,13]$, FaCT [14], InstanceStore [15] and Racer [16]. Some results of the tests are presented in Table 4 ( $\mathrm{FaCT}$ is not included in the table because it lacks support for ABox; also we did not succeed to automatically populate ABox of InstanceStore).

\begin{tabular}{|c|c|c|}
\hline $\begin{array}{c}\text { Number of } \\
\text { concepts in } \\
\text { taxonomy }\end{array}$ & $\begin{array}{c}\text { Processing and } \\
\text { post-processing } \\
\text { time [s] }\end{array}$ & $\begin{array}{c}\text { Signature } \\
\text { generation time } \\
\text { [s] }\end{array}$ \\
\hline 3357 & 72 & $<1$ \\
\hline 82706 & 380 & 12 \\
\hline 184086 & 857 & 115 \\
\hline 545450 & 1386 & 2253 \\
\hline
\end{tabular}

Table 3. Results of experiments for signature generation for pure taxonomies. 


\begin{tabular}{|l|c|c|c|}
\cline { 2 - 4 } \multicolumn{1}{c|}{} & \multicolumn{3}{c|}{ Ontology loading time [s] } \\
\hline \multicolumn{1}{c|}{ Size of ABox } & 400 & 1000 & 3800 \\
\hline Jena & 1 & 22 & - \\
\hline Racer & 3 & 4 & 5 \\
\hline KASEA & 43 & 122 & 465 \\
\hline & Query processing time [s] \\
\hline \multicolumn{1}{|c|}{ Size of ABox } & 400 & 1000 & 3800 \\
\hline Jena & 6 & 250 & - \\
\hline Racer & 58 & - & - \\
\hline KASEA & $<1$ & $<1$ & 1 \\
\hline
\end{tabular}

Table 4. Results of experiments. Hyphens indicate that the activity did not be completed in 2 hours.

The main difference between the analyzed reasoning tools is in time of loading an ontology (ABox Tells). As expected, time of loading an ontology is longer for KASEA. In return we obtain a very short response time. Scalability is also much better for KASEA. While Racer was unable to answer a query after 1000 individuals have been loaded, KASEA processed the same query for 11000 individuals in 1.4 seconds (this specific result is not shown in Tab. 4).

5. Further development. Our present work focuses on overcoming some limitations of KASEA. The most important ones are: restriction of use of $\exists R$.C concepts in queries and shortcomings of neighborhood update.

Although the first limitation may seem inherent for Cartographic Approach, it may be overcome by using signatures with variable length. Although the range of function $s$ becomes theoretically infinite, very long signatures are assigned to complex concepts that are very unlikely to appear in queries. The second limitation stems from complexity of the neighborhood update mechanism and from the fact that it is not fully OWA-compliant [17].

Moreover, we are gradually extending KASEA capabilities in order to support such constructs as cardinality constraints and symmetric, transitive and functional roles, so

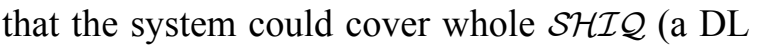
dialect that OWL-DL is based on). We also extend signature analysis on roles to support role hierarchies analogously to concept hierarchies. Other research topics focus on integration of knowledge from different ontologies [18] and on reasoning over ontologies that are not fully trustworthy (e.g. they come from knowledge sources that are not fully trusted [19]).

\section{Bibliography}

1. Baader F. A., McGuiness D. L., Nardi D., PatelSchneider P. F. The Description Logic Handbook: Theory, implementation, and applications, Cambridge University Press, 2003.

2. OWL - Web Ontology Language Guide, W3C, www/w3.org/2004/OWL

3. W3C Semantic Web Activity, http://www.w3.org/2001/sw

4. Berners-Lee, T., Hendler, J., Lassila, O. „,The Semantic Web". Scientific American, May 2001.

5. http://www.pips.eu.org

6. Goczyła, K., Grabowska, T., Waloszek, W., Zawadzki, M. „Problematyka zarządzania wiedzą w systemach typu e-health”. In: „Inżynieria oprogramowania - Nowe wyzwania", Ed. J. Górski, A. Wardziński, WNT 2004, Chap. XXVI.

7. Goczyła K., Grabowska T., Waloszek W., Zawadzki M. "The Cartographer Algorithm for Processing and Querying Description Logics Ontologies". LNAI, Vol.3528, 2005, pp. 163-169.

8. Minsky, M. „A Framework for Representing Knowledge". In: The Psychology of Computer Vision. Ed. P.H.Winston, McGraw-Hill, New York, 2005, pp. 211-277.

9. Bechhofer S.: "The DIG Description Logic Interface: DIG/1.1”, Univ. of Manchester, 2003.

10. Bryant, R. E. "Graph-based algorithms for Boolean function manipulation", IEEE Transaction on Computers, 1986.

11. DIGUT Interface, V1.3. KMG@GUT, 2005, http://km.pg.gda.pl/km/DIGUT_Interface_1.3.pdf

12. A Semantic Web Framework for Java, http://jena.sourceforge.net

13. D. Reynolds: "Jena 2 Inference support", 2004, http://jena.sourceforge.net/inference/index.html

14. I. Horrocks: "FaCT Reference Manual v1.6", 1998, http://www.cs.man.ac.uk/ horrocks/FaCT

15. S. Bechhofer, I. Horrocks, D. Turi: "Implementing the Instance Store", available at CEUR http://sunsite.informatik.rwthaachen.de/Publications/CEUR-WS/Vol-115

16. V. Haarslev, R. Möller: "RACER User's Guide and Reference Manual", September 17, 2003, http://www.cs.concordia.ca/ haarslev/racer/racermanual-1-7-7.pdf

17. Goczyła K., Waloszek W. „Topologiczna analiza ontologii opartych na logice opisowej”. In: „Bazy danych - modele, technologie, narzędzia. Analiza danych i wybrane zastosowania", Ed. S. Kozielski, B. Małysiak, P. Kasprowski, D. Mrozek. WKŁ, 2005, Chap. 23.

18. Goczyła K., Grabowska T. „Przetwarzanie zapytań w rozproszonej bazie wiedzy opartej na logice opisowej", ibid., Chap. 24.

19. Goczyła K., Zawadzki M. „Przetwarzanie i wnioskowanie $\mathrm{z}$ wiedzy o różnym poziomie zaufania”, ibid., Chap. 25. 